\title{
OSCILAÇÃO DA PRESSÃo EM TAHITI E SUA RELAÇÃO COM A VARIAÇÃO dO VENTO ZONAL NO BRASIL
}

\author{
SANTOS, André Gonçalo - andregs9@yahoo.com.br \\ DEHA / UFC - Universidade Federal do Ceará \\ MOLION, Luiz Carlo Baldicero - molion@ufal.br \\ ICAT / UFAL - Universidade Federal de Alagoas
}

\begin{abstract}
RESUMO
O objetivo deste estudo foi analisar o grau de associação entre a oscilação da pressão atmosférica ao nível do mar (PNM) em Tahiti e a oscilação do vento zonal (VZ) em $850 \mathrm{mbno}$ Brasil. Para esse propósito, foi utilizado um conjunto de dados de reanálise do NCEP/NCAR de PNM e VZ, para um período que compreende duas fases da Oscilação Decadal do Pacífico (ODP), uma fase fria (1948-1976) e outra quente (1977-1998). Primeiramente, foi desenvolvida uma série temporal do Índice de PNM Padronizado em Tahiti (IPPT), que foi correlacionado com a variável acima mencionada sobre o Brasil. O software GrADS foi usado para confeccionar os mapas de coeficientes de correlação entre o IPPT e VZ. A correlação espacial foi feita para a variável em cada fase (fria e quente) da ODP separadamente, e foram encontrados valores de coeficientes de correlações diretas e inversas entre o IPPT e o VZ, superiores a 0,5 em algumas regiões, com nível de significância estatística de até $99 \%$ em ambas as fases da ODP. Correlações adiantadas de até 5 meses também foram feitas entre o IPPT e o VZ, e os resultados sugeriram que o IPPT se adequou como bom previsor do VZ com até 5 meses de antecedência, com significância estatística acima dos $98 \%$ para algumas regiões da área de estudo. Deste modo, concluiu-se que é inegável a influência da oscilação da pressão do oceano Pacífico (Ilha de Tahiti) sobre a variação do vento zonal no Brasil, e tal informação ajudará no processo de planejamento das atividades agrícolas e sociais.
\end{abstract}

Palavras-Cahves: Significância estatística; Vento zonal; Previsibilidade.

OSCILLATION OF PRESSURE IN TAHITI AND ITS RELATION WITH OF THE ZONAL WIND VARIATION IN BRAZIL

ABSTRACT: The objective of this study was to analyze the degree of association between the oscillation of atmospheric sea level pressure (SLP) in Tahiti and zonal wind (ZV) in $850 \mathrm{mb}$ in Brazil. For this purpose, we used a set of reanalysis data from NCEP / NCAR of SLP and ZV, for a period that includes two phases of the Pacific Decadal Oscillation (PDO), a cold phase (1948-1976) and one hot (1977 -1998). Firstly, was developed a temporal series of Index of Standardized SLP on Tahiti (SIPT), which was correlated with the variable mentioned above about Brazil. The GrADS software was used to confection the maps of correlation coefficients between the SIPT and ZV.The spatial correlation was made to the variable in each phase (cold and hot) of PDO separately, and were found values of correlation coefficients between the SIPT and ZV direct and inverse, superiors than 0.5 in some regions, with a significance level statistical up to $99 \%$ in both phases of PDO. Advance correlations of up to 5 months were also made between the IPPT and the VZ, and the results suggested that the IPPT can be a good predictor of the VZ with up to 5 months in advance, with statistical significance above $98 \%$ for some parts of the area study. Thus, it was concluded that there is an undeniable influence of pressure fluctuation of the Pacific Ocean (Tahiti Island) on the variation of zonal wind in Brazil, and such information will assist in the planning process of agricultural and social activities.

Keywords: Statistical significance; Zonal wind; Predictability.

\section{INTRODUÇÃO}

O Brasil encontra-se em uma região individualmente favorável em termos de teleconexões atmosféricas relacionadas ao fenômeno EI Niño Oscilação Sul (ENOS) e a Oscilação Decadal do Pacífico (ODP), que segundo Mantua (1997) se caracteriza por uma oscilação da temperatura da superfície (TSM) do Oceano Pacífico, com variabilidade mais longa que a dos episódios ENOS, e escala temporal média de 50 a 60 anos, com duas fases, uma fase fria e outra quente, com duração de 20 a 30 anos cada fase. As variações de TSM associadas à ODP interagem com a atmosfera, sobre o Pacífico, e muda o campo de pressão, e consequentemente a circulação dos ventos zonais.

O vento zonal é um dos mecanismos da atmosfera que tem por função o transporte de energia de uma região a outra. Wauhg e Funatsu(2003) mostraram que nas regiões equatoriais os ventos zonais $(850 \mathrm{mb}$ ) são favoráveis à difusão de energia (Oceano Atlântico e Oceano Pacífico). Matsumoto (1973) em sua pesquisa mostrou que existe uma grande relação entre a precipitação e a velocidade do vento. E esta relação está ligada com o transporte de calor latente. Costa (1976) em seu estudo descreve que a alteração na direção do vento nos baixos níveis, está associada à ocorrência de precipitação. E segundo Yamazaki (1972) e Ramos (1974), a oscilação do vento zonal pode causar uma variação nas características climatológicas em baixos níveis. 
Dessa forma, a variação do campo de pressão ao nível médio do mar (PNMM) no Pacífico equatorial, associado aos fenômenos ENOS e ODP, tem condicionado de maneira contundente via teleconexões, a variação dos ventos zonais sobre o Brasil, o que por sua vez, altera o transporte de energia e consequentemente condiciona alterações no regime pluviométrico.

Contudo, pensou-se em explorar, neste estudo, a série da PNMM padronizada da região de Tahiti localizada no oceano Pacífico Tropical Sus, devido a sua proximidade com o Brasil, e procurar estabelecer possíveis relacionamentos de sua variabilidade com as variações decadais do Vento Zonal. A série também foi explorada no que se refere ao seu caráter preditivo correlacionado ao vento zonal, com o objetivo de auxiliar no planejamento das atividades socioeconômicas.

\section{METERIAIS E MÉTODOS}

\section{Materiais Utilizados}

Para o estudo da variação da pressão em Tahiti e sua relação com o vento zonal no Brasil, foi utilizado um conjunto de dados de reanálises disponível no ESRL/PSD/NOAA (Earth System Research Laboratory, Physical Science Diveision, National Oceanic Administration Atmosferic) de Pressão ao Nível do Mar (PNMM) e vento zonal (VZ) ao nível de $850 \mathrm{mb}$ em pontos de grade de $2,5^{\circ} \times 2,5^{\circ}$, de Abril a Julho para um período de 1950 a 1998, correspondente a duas fases da Oscilação Decadal do Pacífico, uma fria (1948 a 1976) e a outra quente (1977 a 1998). Os dados foram adquiridos no site do www.cdc.noaa.gov e são descritos com maiores detalhes por (KALNAY et al., 1996).

\section{Área de Estudo}

A área de estudo compreende todo território nacional Brasileiro, que está localizado entre os paralelos de $5^{\circ} 16^{\prime}$ de latitude norte e $33^{\circ} 44^{\prime}$ de latitude sul, e entre os meridianos de $34^{\circ} 47^{\prime}$ e 73059' de longitude oeste, com uma área total de $8.514 .876 \mathrm{~km}^{2}$.

Os principais climas do Brasil são: equatorial, semiárido, tropical, tropical de altitude e subtropical.

\section{Elaboração do Índice de Pressão Padronizadaem Tahiti (IPPT)}

Na elaboração do IPPT, foram utilizados dados de reanálises de pressão ao nível médio do Mar, de Janeiro a Dezembro no período de 1948 a 2009, disponível no ESRL/PSD/NOAA, para o domínio geográfico de Tahiti a maior ilha da Polinésia Francesa, localizada na coordenada (lat: $17^{\circ} 40^{\prime} 0^{\prime \prime}$ Se lon: $149^{\circ} 27^{\prime} 0^{\prime \prime}$ W) que fica na região central do Pacífico Tropical Sul.

O software utilizado no cálculo estatístico na elaboração do IPPT foi à planilha calc do openoffice/broffice. E na elaboração dos campos, foi utilizado o software meteorológico Grid Analysis and Display System (GrADS)na correlação do índice IPPT com as variáveis em estudo. Após a criação do índice, utilizamos uma ferramenta interativa do site www.cdc.noaa.com, para realização das correlações com a variável em estudo. 


\section{Cálculo Estatístico}

Formulação matemática:

$$
I P P T=\frac{X_{i j}-\bar{\mu}_{j}}{\sigma_{j}}
$$

Na qual: O IPPT é a padronização dos dados, em que $X_{i j}$ é o valor encontrado em um determinado ano (i) no mês (j).

$$
\bar{\mu}_{j}=\frac{1}{n} \sum_{i=1}^{n} x
$$

Onde $\bar{\mu}_{j}$ é o valor médio de um dado mês (j).

$$
\sigma_{j}=\sqrt{\frac{\sum_{i}^{n}(x-\bar{\mu})^{2}}{n-1}}
$$

É $\sigma_{j}$ é o desvio padrão médio para um dado mês (j), do período de estudo.

\section{Coeficiente de Correlação de Pearson}

Segundo Schultz e Schultz (1992) um dos métodos mais conhecido para medir a correlação entre duas variáveis é o Coeficiente de Correlação Linear de Pearson, estudado por Francis Galton e Karl Pearson em 1897. A hipótese básica para o emprego deste coeficiente é de que o relacionamento entre as duas variáveis sejam linear.

O coeficiente de Pearson é conhecido como correlação ordinária ou correlação linear. Ele é uma ferramenta simples e eficiente para estimar o grau de relacionamento entre duas variáveis quaisquer. Os valores das correlações estão limitados no intervalo de -1 e 1 .

A formulação utilizada para calcular o coeficiente de correlação de Pearson é:

$$
r=\frac{\sum_{i=1}^{n}\left(x_{i}-\bar{x}\right) *\left(y_{i}-\bar{y}\right)}{\sqrt{\sum_{i=1}^{n}\left(x_{i}-\bar{x}\right)^{2} * \sum_{i=1}^{n}\left(y_{i}-\bar{y}\right)^{2}}}
$$

Para qual, "i" é o ano inicial, " $n$ " o ano final.

$\bar{x} \mathrm{e} \bar{y}$ são as médias aritméticas das variáveis $x_{i}$ e $y_{i}$.

A partir disso, se as duas variáveis correlacionadas apresentarem uma reta crescente (decrescente), a correlação é positiva (negativa), ao maior (menor) grau de associação, é atribuído o valor $1(-1)$.

A correlação apenas dá uma noção do grau de relacionamento das variáveis, porém não implica uma relação de causa e efeito.

Foi utilizado um teste de hipótese que qualifica os valores das correlações e determina a significância estatística dos resultados encontrados, ou seja, o nível de significância dos coeficientes de correlação. Essa técnica determina o quão significativos são os resultados. A tabela 1 mostra os resultados do cálculo dos níveis de significância em relação aos valores das correlações e o grau de liberdade (nesse caso, os anos).

A tabela explica, por exemplo, que dado um grau de liberdade de 28 (correspondente aos anos da fase fria da ODP de 1948 a 1976) anos, os testes de nível de significância indica que, para um coeficiente de correlação com valor em módulo de 0,44, tem-se uma significância de $99 \%$ de chance que as variáveis realmente estejam correlacionadas. Ou seja, apenas $1 \%$ de chance que a hipótese nula se confirme, ou seja, que as variáveis não estejam relacionadas. 
TABELA1: $\quad$ Graus de liberdade e os níveis de significância dos coeficientes de

\begin{tabular}{|c|c|c|c|c|c|}
\hline $\begin{array}{l}\text { Graus de } \\
\text { Liberdade }\end{array}$ & 0,90 & 0,95 & 0,98 & 0,99 & 1,00 \\
\hline 20 & 0,319 & 0,377 & 0,441 & 0,512 & 0,557 \\
\hline 21 & 0,310 & 0,367 & 0,431 & 0,500 & 0,545 \\
\hline 22 & 0,302 & 0,358 & 0,421 & 0,489 & 0,533 \\
\hline 23 & 0,295 & 0,350 & 0,411 & 0,479 & 0,522 \\
\hline 24 & 0,289 & 0,343 & 0,403 & 0,469 & 0,512 \\
\hline 25 & 0,282 & 0,336 & 0,395 & 0,460 & 0,503 \\
\hline 26 & 0,276 & 0,329 & 0,387 & 0,451 & 0,493 \\
\hline 27 & 0,270 & 0,322 & 0,380 & 0,443 & 0,485 \\
\hline 28 & 0,265 & 0,316 & 0,373 & 0,436 & 0,476 \\
\hline 29 & 0,260 & 0,311 & 0,366 & 0,428 & 0,469 \\
\hline 30 & 0,255 & 0,305 & 0,360 & 0,421 & 0,461 \\
\hline 40 & 0,219 & 0,264 & 0,312 & 0,366 & 0,402 \\
\hline
\end{tabular}

\section{RESULTADOS E DISCUSSÕES}

A figura 1 tem por objetivo mostrar o padrão médio do vento zonal (850mb) sobre o Brasil, e o que se constatou foram duas regiões distintas, a primeira é a região tropical, com ventos zonais de leste e intensidade variando de 7 a $30 \mathrm{~km} / \mathrm{h}$ aproximadamente, como se constata nas capitais (Fortaleza, São Luís, Belém e Macapá) com valores (30 km/h) máximos, o que está de acordo com a velocidade obtida pela FUNCEME em Fortaleza. E a segunda é a região subtropical, com ventos de oeste, e intensidade em tornos dos 7,2 km/h. Contudo, vale salientar, que cada região está submetida a sistemas báricos sinóticos distintos, o que explica o sentido de onde os ventos (zonal) sopram e disparidade na intensidade dos ventos nestas duas regiões.

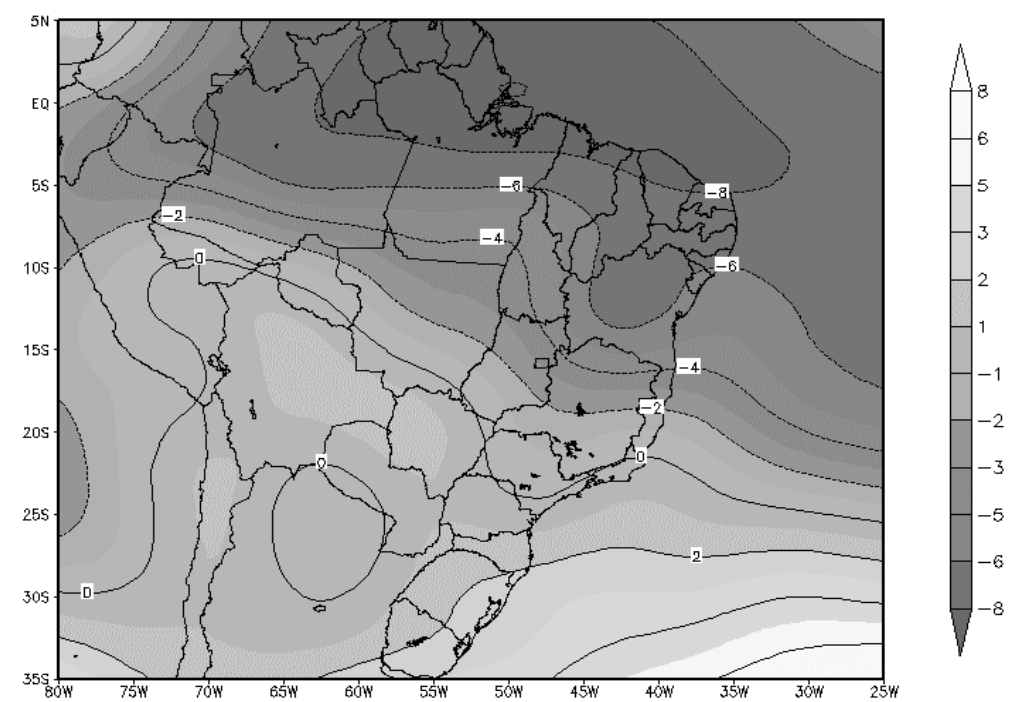

Figura 1: Média anual do Vento Zonal em 850mb (m/s), para um período de 1948 a 2011. 
Foi realizado um diagnóstico com vento zonal na fase fria (1948 a 1976) e quente (1977 a 1998) da ODP sobre o território Brasileiro, correlacionado com o IPPT (Figura 2).

A Figura 2 é uma representação gráfica da série temporal do IPPT para um período de 59 anos, com uma média móvel de 11 meses, para filtrar o ciclo anual. Observa-se que essa série apresenta um padrão interanual de variação com uma similaridade ao Índice de Oscilação Sul (IOS). Em geral, os desvios positivos (negativos) estão associados a eventos La Niña (El Niño).

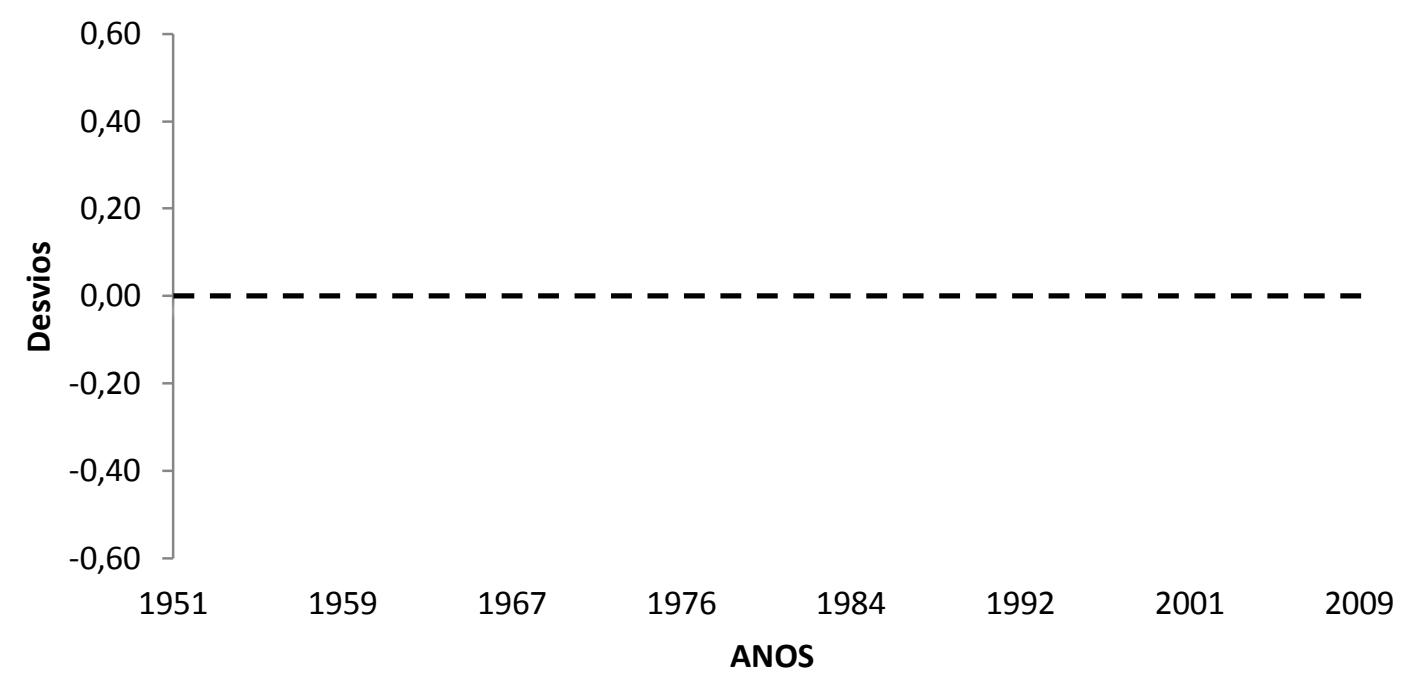

Figura 2: $\quad$ Índice de Pressão Padronizadaem Tahiti (IPPT) para o período de 1951 a 2009, com a média móvel de 11 meses.

As correlações do índice de pressão Padronizadaem Tahiti (IPPT) com o vento zonal foi elaborada para o período AMJJ na fase fria (1948 a 1976) e quente (1977 a 1998) da ODP e seus resultados são mostrados a seguir.

\section{Analise}

A Figura 3expõe os resultados da correlação espacial entre o IPPT e o campo da componente zonal do vento no nível de $850 \mathrm{mb}$. E constatou-se na FF da ODP (Figura 3a), coeficientes de correlações com valores entre 0,2 e 0,4, com níveis de significância estatística entre $90 \%$ e $95 \%$, sobre o Amapá, norte do Pará e no extremo norte da região Nordeste. Isso sugere que o vento zonal tenha se tornado mais intenso nessas áreas durante a $\mathrm{FF}$, decorrente do aumento de pressão (La Niña) em Tahiti, admitindo que nas fases frias da ODP, há uma maior predominância dos fenômenos La Niña (MOLION, 2005). Notou-se, também, valores negativos sobre Rondônia, sul do Pará e Mato Grosso, que corresponde um enfraquecimento do vento zonal. Nas regiões Sul e Sudeste, a componente zonal também apresentou valores positivos, sugerindo a intensificação do vento zonal nessas áreas. Já na FQ da ODP (Figura 3b), é constatado um núcleo de valores positivos e coeficiente de correlação entre 0,4 a 0,6 com níveis de significância entre $95 \%$ e $99 \%$, porém, posicionado mais para o interior do continente entre as latitudes $5 \mathrm{~S}$ e $5 \mathrm{~N}$, possivelmente associados com o deslocamento do ramo descendente da célula de Walker. Fato notório foi observado sobre a Região Sul, particularmente o Rio Grande do Sul, que apresentou uma situação inversa ao da FF, com núcleos de até $-0,7$, indicando que uma redução da PNMM em Tahiti (El Niño) esteve associada ao aumento da intensidade da componente zonal do vento nesta região. Dessa forma, é 
evidente que nos setores onde os coeficientes são superiores a 0,2 (em modulo), as correlações são altíssimas entre a variação da pressão e o vento zonal.

Tabela 2: Graus de liberdade e nível de significância

estatísticana FF.

Significância

Estatística

\begin{tabular}{cc}
\hline S.E & G.L (28) \\
$90 \%$ & 0,265 \\
$95 \%$ & 0,316 \\
$98 \%$ & 0,373 \\
$99 \%$ & 0,436 \\
$100 \%$ & 0,476 \\
\hline
\end{tabular}

Tabela 3: Idem a tabela 2, só que na FQ.

\begin{tabular}{cc}
\multicolumn{2}{c}{$\begin{array}{c}\text { Significância } \\
\text { Estatística. }\end{array}$} \\
\hline S.E & G.L (21) \\
$90 \%$ & 0,310 \\
$95 \%$ & 0,367 \\
$98 \%$ & 0,431 \\
$99 \%$ & 0,500 \\
$100 \%$ & 0,545 \\
\hline
\end{tabular}
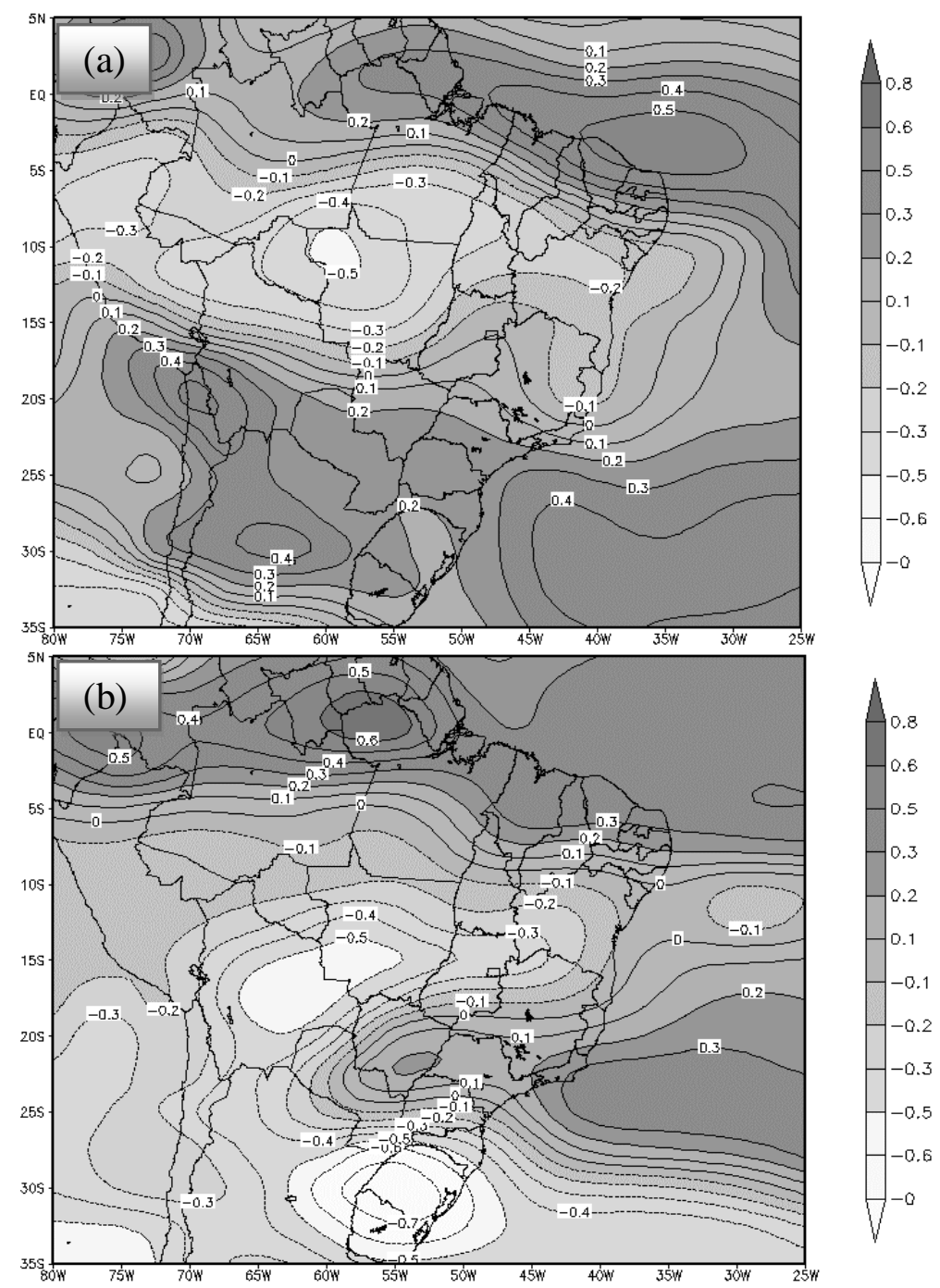

Figura 3: Coeficientes de Correlação do vento zonal em $850 \mathrm{mb}$ com o IPPT na fase fria (a) e fase quente (b) da ODP.

\section{Coeficiente Adiantado}

A importância da correlação adiantada está no tempo necessário que se obtém para se estabelecer um planejamento consistente, na agricultura e em outras atividades sociais. $\mathrm{Na}$ analise da correlação espacial do IPPT avançado em cinco meses na FF da ODP (figura 4a), constatou-se valores de coeficientes de correlações diretas e elevadas no extremo norte do Brasil, chegando a 0,6 (100\%de significância estatística)(tabela 4) no norte do estado do Amazonas, indicando que se houver um aumento da pressão em Tahiti, e se esse aumento persistir existirá uma grande probabilidade de que cinco meses depois, a intensidade dos ventos zonais aumentará nesta região, proporcionando assim um maior (menor) transporte de energia, o que consequentemente afetará o regime pluviométrico desta área. Já no estado de Rondônia, foram constatadas correlações inversas, indicando uma situação contrária ao do estado do Amazonas.

$\mathrm{Na} F Q$ (figura 4b), os coeficientes foram positivos, expressando uma correlação direta na região Norte e Nordeste do Brasil, com nível de significância estatística acima dos 95\%, como no estado do Ceará $(0,6)$, Amapá $(0,5)$ e Amazonas $(0,4)$. Tais estimações refletem a influência direta dos fenômenos ENOS e ODP, tanto no campo do vento zonal quanto na 
precipitação, como é citado no estudo de (SANTOS 2006). Na região Sul, Sudeste e Centro Oeste, o sinal foi oposto ao da região Norte e Nordeste, expressando uma situação totalmente contrária, com níveis de significâncias superiores a $90 \%$, com o maior grau de associação no oeste do Mato Grosso do Sul. Contudo, ficou evidente nesta analise, que além dos altos valores de correlações adiantada, as duas fases da ODPapresentaram padrões distintos no Sul, Sudeste e Centro Oeste do Brasil, proporcionando coeficientes maiores no Noroeste da região Norte na FF e no norte do Nordeste na FQ.

Tabela 4: Graus de liberdade e nível de significância

estatísticana FF.

\begin{tabular}{cc}
\multicolumn{2}{c}{$\begin{array}{c}\text { Significância } \\
\text { Estatística. }\end{array}$} \\
\hline S.E & G.L (28) \\
$90 \%$ & 0,265 \\
$95 \%$ & 0,316 \\
$98 \%$ & 0,373 \\
$99 \%$ & 0,436 \\
$100 \%$ & 0,476 \\
\hline
\end{tabular}
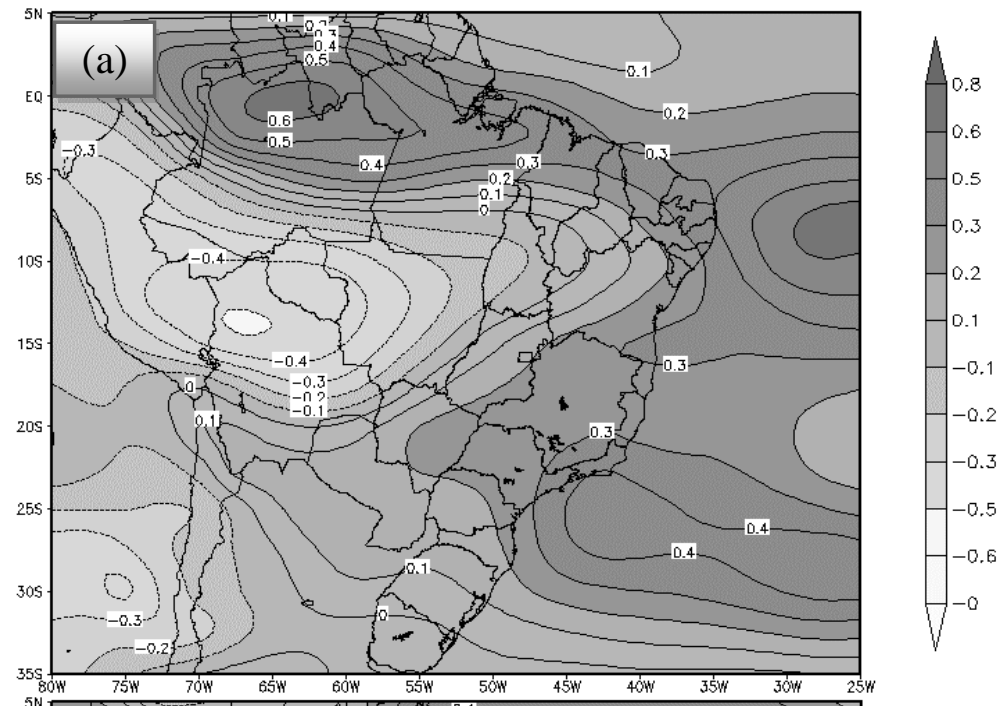

Tabela 5: Idem a tabela 4, só que na FQ.

\begin{tabular}{cc}
\hline \multicolumn{2}{c}{$\begin{array}{c}\text { Significância } \\
\text { Estatística. }\end{array}$} \\
\hline S.E & G.L (21) \\
$90 \%$ & 0,310 \\
$95 \%$ & 0,367 \\
$98 \%$ & 0,431 \\
$99 \%$ & 0,500 \\
$100 \%$ & 0,545 \\
\hline
\end{tabular}

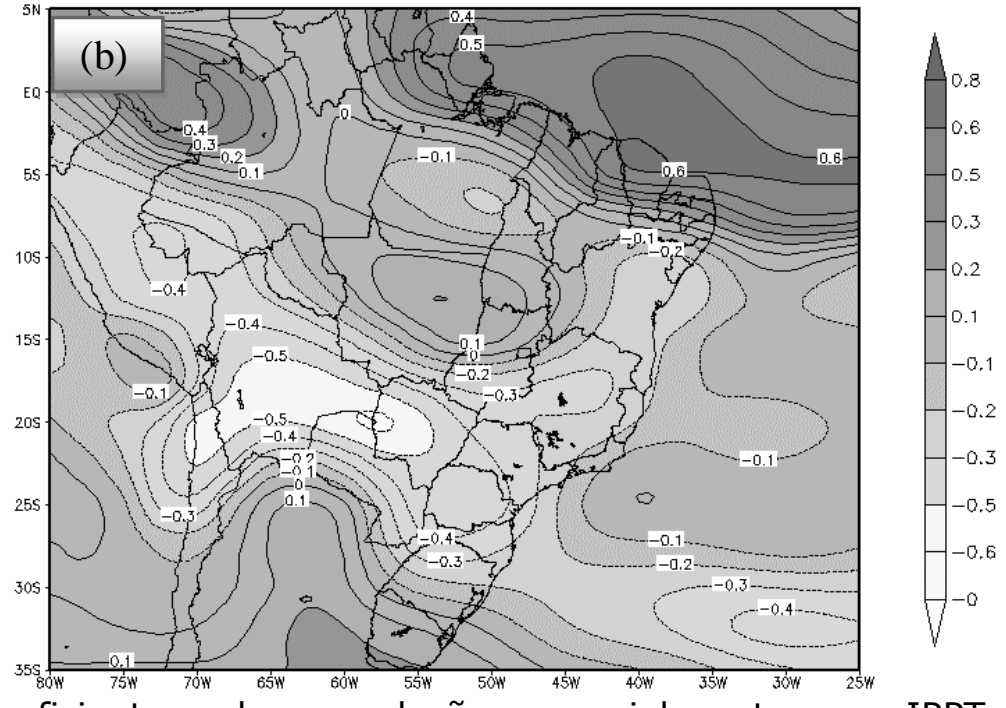

Figura 4: Coeficientes de correlação espacial entre 0 IPPT, adiantado em cinco meses, e o vento zonal $(\mathrm{m} / \mathrm{s})$ em $850 \mathrm{mb}$ para as fases (a) fria (1948 a 1976) e (b) quente (1977 a 1998) da ODP.

\section{CONCLUSÕES}

Neste estudo, procurou-se avaliar a correlação entre a variação da pressão na Ilha de Tahiti, localizada no Pacífico Tropical, e o vento zonal sobre o Brasil. Contudo, concluímos que:

O Brasil é dividido em duas regiões distintas no campo do vento zonal, uma é a região tropical com ventos de leste e com velocidade que atinge $30 \mathrm{~km} / \mathrm{h}$, e a outra subtropical com ventos de oeste e intensidade em tornos dos $7,2 \mathrm{~km} / \mathrm{h}$. 
As correlações feitas entre o IPPT e o campo do vento zonal, foram significativas em variais regiões do Brasil, como na região Norte, norte do Nordeste, Centro Oeste, Sul e Sudeste, mostrando níveis de significância estatística acima dos 98\%, com valores de correlações absolutos mais expressivos na FQ da ODP. Os valores aqui registrados indicam uma associação extremamente estreita entre o campo de pressão em Tahiti e a variação do vento zonal no Brasil, o que estão ligados ao transporte de energia e variação dos índices pluviométricos.

O IPPT avançado em cinco meses mostrou valores elevados de correlações diretas e inversas, em diferentes regiões do Brasil, tanto na FF quanto na FQ e com significância estatísticas superiores a 95\%, sugerindo que quando houver um aumento (redução) de pressão em Tahiti (La Niña) e esta variação persistir, cinco meses posteriormente a análise, o índice mostrará que haverá um aumento (redução) na intensidade dos ventos zonais no Brasil, principalmente no extremo norte do Brasil e no estado do Mato Grosso, Paraná e São Paulo.

\section{REFERÊNCIAS BIBLIOGRÁFICAS}

COSTA, M. N. Oscilação no Vento Zonal e na Temperatura Estratosfera, São José dos Campos, 1979. 59 f. INPE, 1976. 59 p. (Dissertação) - Programa de Pós-Graduação em Ciência Espacial e da Atmosfera. Instituto Nacional de Pesquisas Espaciais, Porto Alegre, São Paulo. 1976.

ESRL/PSD/NOAA - Earth System Research Laboratory - Serving Society Through Science. http://www.esrl.noaa.gov

KALNAY, E.; KANAMITSU, M; KISTLER, R.; COLLINS, W.; DEAVEN, D.; GANDIN, L.; IREDELL, M.; SAHA, S.; WHITE, G.; WOOLEN, J.; ZHU, Y.; CHELLIAH, M.; EBISUZAKI, W.; HIGGINS, W.; JANOWIAK, J.; MO, K. C.; ROPELEWSKI, C.; WANG, J.; RENYNOLDS, R.; JENNE, R.; JOSEFH, D.; The NCEP/NCAR 40-year Reanalysis Project. Bulletin of the American Meteorological Society, v. 77, n.3, p. 437-471, 1996

MOLION, L. C. B. Aquecimento Global. El Niños, Manchas Solares, Vulcões e Oscilação Decadal do Pacífico. Climanalise, 2005.

MANTUA, N.J.;HARE,S.R.; ZHANG, Y.;WALLACE, J.M.; FRANCIS, R.C. A Pacific interdecadal climate oscillation with impacts on salmon production. Bulletin of the American Meteorological Society, 78, p. 1069-1079, 1997.

MATSUMOTO, S. - Lower troposphere Wind speed and precipitation activity. J. Met. Soc. of Japan, 51: 101-107, 1973.

SANTOS, D. M.; ROCHA, E. J. P.; RIBEIRO, J. B. M.; ROLIM, P. A.M. Influência dos Fenômenos El Niño e La Niña na Precipitação do Estado do Amazonas. In: II Workshop de Avaliação Técnica e Científica da Rede CTPetro Amazônia, 2006, Manaus.

SCHULTZ, D. P.; SCHULTZ, S. E. História da psicologia moderna. 16. ed. São Paulo. Cultrix.p.439, 1992.

RAMOS, R.P.L. Precipitation characteristics in the Northeast Brazil dry region. Atmospheric Science Paper. n. 224, Colo. State Univ., Ft. Collins, Colorado, p. 56, 1974.

WAUGH, D.W., FUNATSU, B.M. Intrusions into the Tropical Upper Troposphere: ThreeDimensional structure and accompanying ozone and OLR distributions. J. Atmos. Sci.,v. 60, p. 637-653, 2003.

YAMAZAKI, Y. - "Estudo teóricos e sinóticos dos distúrbios tropicais". INPE - LAFE 624, 1972. 